\title{
Designing of high-resolution photoresists: use of modern NMR techniques for evaluating lithographic performance
}

\author{
DEBMALYA ROY ${ }^{\dagger}, P$ K BASU ${ }^{\dagger}, P$ RAGHUNATHAN ${ }^{*}$ and S V ESWARAN* \\ St. Stephen's College, University of Delhi, Delhi 110 007, India \\ ${ }^{\dagger}$ Solid State Physics Laboratory, Lucknow Road, Delhi 110 054, India \\ ${ }^{\ddagger}$ Fluorosis Research Foundation, 34, I.P. Extension, Delhi 110 092, India
}

MS received 12 August 2003; revised 8 April 2004

\begin{abstract}
Applications of improved 1-D/ 2-D NMR spectroscopic techniques have been reviewed for quantitatively estimating the incorporation of different monomers and degree of linearity in resin microstructure. Comparison of the NMR data with those from lithography leads to a distinct correlation between resin microstructure and lithographic performance. A novel photoresist mechanism is proposed in a positive photoresist; also, using modern NMR techniques, the crosslinking mechanism in a negative photoresist has been studied.
\end{abstract}

Keywords. Photoresists; lithographic performance; novolac resins; NMR.

\section{Introduction}

Photoactive compounds blended with polymeric material, solvents and certain other additives are referred to as photoresists (Turner and Daly 1988; Wallraff and Hinsberg 1999) and are used in printed electronic circuits at the micron or sub-micron level. Patterns are generated based on changes in the physical and chemical properties of the exposed and unexposed photoresist layers. In positive photoresists, the exposed part becomes soluble in the alkaline developer, whereas in negative photoresists the exposed part is rendered insoluble in the developer, which is usually an organic solvent like xylene (Blevins et al 1987; Roy et al 2002).

Diazonaphthoquinone (DNQ) esters and novolac resins dissolved in ethyl cellosolve acetate (ECA), or the more environmental friendly ethyl lactate, along with certain additives serve as positive photoresists. DNQ undergoes the Wolff rearrangement in presence of light and gets converted to the unstable ketocarbene, which hydrolyzes further to the more stable indene carboxylic acid (figure 1) (Reiser et al 1996). The increased solubility of the positive photoresists on exposure has been traditionally attributed to the formation of indene carboxylic acid. Hanabata et al (1989) proposed that in the unexposed region of positive photoresists, diazonaphthoquinone underwent azo-coupling at the free para position of $m$-cresol incorporated in novolac resin and the azo-coupled products increased the molecular weight of novolac resin, thereby rendering it more insoluble in base.

The two main components of a negative photoresist are aromatic azides/bis-azides and cyclized rubber, along with

*Author for correspondence organic solvent and certain additives. The hydrogen abstraction and/or insertion reaction of nitrene, generated from azide during photolysis, produces a crosslinked network of polymers in the exposed part and thus increases the molecular weight of the resin compared to the unexposed part, consequently changing in solubility characteristics (figure 2). Exploiting this change in the physical property on exposure followed by development often using an orga-

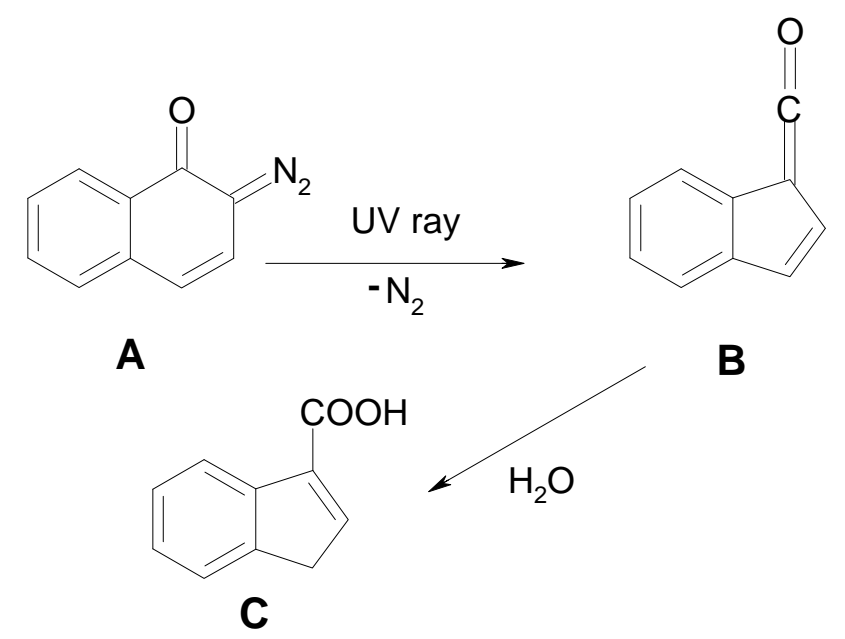

Figure 1. The Sues mechanism: ' $A$ ' is 2, 1-diazonaphthoquinone; ' $\mathrm{B}$ ' is the ketene intermediate and ' $\mathrm{C}$ ' is indene-1-carboxylic acid. On photolysis the diazo compound is converted to the ketene via the unstable ketocarbene or oxirene intermediate, which is finally hydrolyzed to indene-3-carboxylic acid. Sues actually proposed the formation of indene-1-carboxylic acid. It was later proved that at or above $0^{\circ} \mathrm{C}$ the isomeric, indene-3-carboxylic acid predominates. 
nic solvent, a pattern can be generated on a substrate (Turner and Daly 1988; Wallraff and Hinsberg 1999; Roy et al 2002). Another new class of negative photoresists introduced is those based on aryl azides and novolac resins (Uchino et al 1991; Hattori et al 1992; Eswaran patent 1996, 1997). These combined features of both negative and positive photoresists yet do not necessitate any change in the instrumental setup (Bähr et al 1997).

The role of novolac resin in promoting the dissolution of photoresists has not been explained, however, in conventional photoresist compositions with a (photoactive compound: novolac) ratio of $5: 1$. It is soon realized that the matrix polymer holds the key for high-resolution geometry (Hanabata et al 1989; Ueno 1998). A proper engineering of the novolac microstructure is, therefore, very crucial for achieving high-performance photoresists. The three important factors are (a) the use of cresol-formaldehyde resins in place of phenol-formaldehyde novolac resin, (b) use of copolymers of $m$-cresol and $p$-cresol with an optimum percentage of around $62 \% \mathrm{~m}$-cresol and $38 \% \mathrm{p}$-cresol, and (c) the use of 'tailor made' 'high ortho' (figure 3B) alternating (figure 3C) and semialternating (figure 3D) novolacs with an optimum polydispersivity $\left(\mathrm{PD}=M_{\mathrm{w}} / M_{\mathrm{n}}\right.$, where $\mathrm{PD}=$ polydispersivity, $M_{\mathrm{w}}=$ weight average molecular weight and $M_{\mathrm{n}}=$ number average molecular weight) ('tandem type' novolac resin) in place of random novolac resin (figure 3A) (Hanabata et al 1989; Fitzgerald 1990; Ueno 1998; Roy et al 2003a). In this work, we have reviewed the use of improved 1-D and 2-D NMR spectroscopic techniques for quantitatively estimating the incorporation of different monomers and the degree of linearity in the resin microstructure.

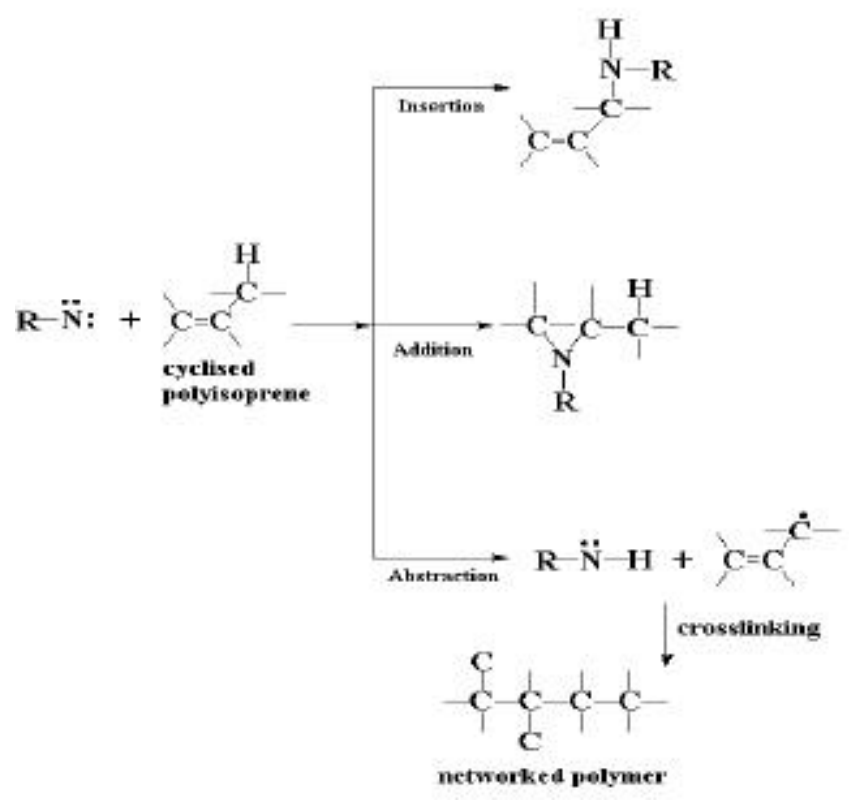

Figure 2. The general crosslinking mechanism leading to negative photoresists.
A deeper insight into the mode of action of photoresists is also equally important for designing high-performance photoresists. However, development of scientific understanding in this area of high technological interest has been rather slow (Zöllinger 1994). It has been proposed that the positive photoresist underwent an azo coupling reaction with DNQ leading to an increase in the molecular weight of the resin, and thereby rendering it more insoluble in the alkaline developer. However, in our NMR experiments we did not find any involvement of free para position of novolac resin in the azo coupling reaction (Roy et al 2003b).

The sharp polarity difference in the exposed and unexposed negative photoresist has been attributed to the increased molecular weight of exposed part due to crosslinked novolac resin with reactive nitrene intermediate, generated from azido photoactive compound (Ueno 1998). However, the site of crosslinking in a novolac resin has remained an open question. It was concluded that the collective effects of attacking the methylenic bridge, aromatic moiety and hydroxyl group of novolac by nitrene resulted in the increase of molecular weight of resin in the exposed negative photoresist (Voigt 1999). In our study no evidence is found for involvement of methylenic hydrogen or aromatic moiety of novolac resin for the crosslinking reaction (Roy et al 2003c).

\subsection{Quantitation of novolac microstructure using $1-D \& 2-D N M R$ data}

Although several common analytical techniques such as FT-IR, UV, GPC, HPLC, and DSC etc have been exploi-

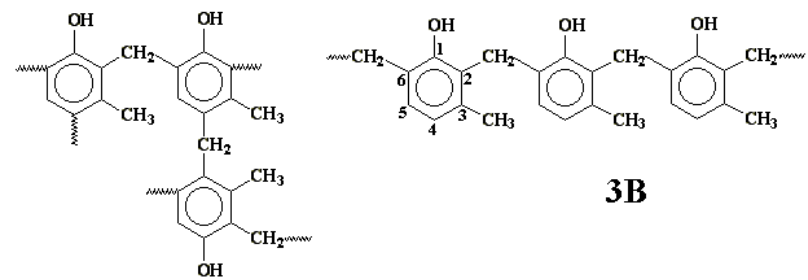

$3 \mathbf{A}$
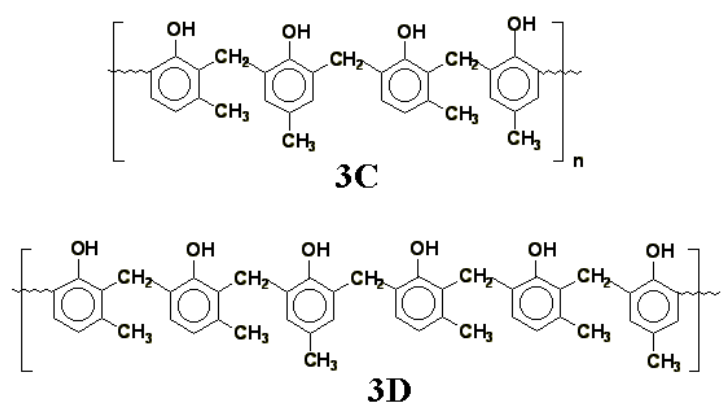

Figure 3. Novolac polymers: ' $3 \mathrm{~A}$ ' is random resin, ' $3 \mathrm{~B}$ ' is 'high ortho' novolac microstructure, ' $3 C^{C}$ ' is alternating $m$-cresol and $p$ cresol based novolac copolymer and '3D' is semialternating novolac copolymer. 
ted for characterizing of novolac resins, they lead only to certain bulk properties. Studies using multidimensional NMR spectroscopy thus become necessary for obtaining several microstructural details, and are exceptionally important for determining the nature and degree of substitution patterns on different ring carbons in novolac resins (Khadim et al 1992; Roy et al 2003a).

Hanabata et al (1989) suggested a substitution index $\left(S_{\mathrm{n}}\right)$ based on the INEPT ${ }^{13} \mathrm{C}$-NMR technique to calculate the available free para position, i.e. degree of 'high ortho' content in the resin. In recent work, $S_{\mathrm{n}}$ has been replaced by the unsubstitution constant $\left(U_{\mathrm{n}}\right)$, the difference being that the latter was calculated using the more sensitive DEPT pulse sequence rather than the one based on the INEPT technique (Khadim et al 1992). The intensities of the ${ }^{13} \mathrm{C}$ NMR spectrum obtained from the INEPT pulse sequence are known to be unreliable for quantitative estimation because of the nuclear Overhauser effect (NOE), which has been corrected by using DEPT pulse sequence. An intensity correlation factor, $I_{\mathrm{n}}$, which is generally considered as 1 , may be first determined by the DEPT ${ }^{13} \mathrm{C}-\mathrm{NMR}$ technique (Khadim et al 1992),

$$
I_{\mathrm{n}}=a_{\mathrm{n}} / a_{5},
$$

where $a_{\mathrm{n}}$ and $a_{5}$ are, respectively, the intensities of the desired position ( 2 or 4 or 6 ) in the phenolic monomer and the position-5 in the precursor.

The unsubstitution index, $U_{\mathrm{n}}$, may then be calculated from DEPT using the formula

$$
U_{\mathrm{n}}=a_{\mathrm{n}} \times 100 / a_{5} \times I_{\mathrm{n}}
$$

where $a_{\mathrm{n}}$ and $a_{5}$ are, respectively, the intensities of the desired position in the resin and position-5.

However, both the earlier NMR spectroscopic strategies suffer from important limitations. Intensities of the ${ }^{13} \mathrm{C}$-NMR spectral absorptions obtained from the INEPT pulse sequence are unreliable for quantitative estimation because of the nuclear Overhauser effect (NOE). Regarding the use of DEPT, as observed by us recently, the pulse sequences experience the problem of unequal J-values. This is due to the effects of polydispersivity, carbon atoms in the same chemical, but different microstructural environments are not associated with the same J-values in DEPT, and therefore, the intensities of ${ }^{13} \mathrm{C}$ NMR would not be totally dependable. We have, therefore, proposed the calculation of $U_{\mathrm{n}}$ values from fully relaxed ${ }^{1} \mathrm{H}-\mathrm{NMR}$ spectra of novolac resins using a combination of 1-D and 2-D NMR techniques (Roy et al 2004a,b).

\section{Experimental}

Novolac copolymers were prepared by both one- and twostep procedures (Kopf 1988; Bähr et al 1997). In the onestep procedure, random polymers were prepared by charging $m$-cresol, $p$-cresol and formaldehyde in $1: 1: 2$ ratios, in presence of an inorganic acid such as conc. hydrochloric acid (1\% molar equivalent of total phenolic components). The mixture was refluxed at $140^{\circ} \mathrm{C}$ using xylene as reaction medium for $1 \mathrm{~h}$. Water formed during polymerization was removed by a Dean-Stark-trap to facilitate further chain growth. The resultant resin was steam distilled at $180^{\circ} \mathrm{C}$ for $1 \mathrm{~h}$ to wash out the unreacted monomer components (table 1).

\begin{tabular}{|c|c|c|c|c|c|c|}
\hline Copolymer & $\begin{array}{c}\text { First } \\
\text { cresolic } \\
\text { monomer 'I' }\end{array}$ & $\begin{array}{c}\text { BHMC } \\
\mathrm{R}=\mathrm{C}_{15} \mathrm{H}_{25-31} \\
\mathrm{R}_{1}=\mathrm{CH}_{3}\end{array}$ & $\begin{array}{c}\text { Second } \\
\text { cresolic } \\
\text { monomer 'II' }\end{array}$ & $\begin{array}{c}\text { Third } \\
\text { cresolic } \\
\text { monomer 'III' }\end{array}$ & Nature & Base solubility \\
\hline SAMPLE 1 & $m$-cresol (M) & & $p$-cresol $(\mathrm{P})$ & $m$-cresol & $\begin{array}{l}\text { "High ortho", semialternating } \\
(\mathrm{P}-\mathrm{M}-\mathrm{M}-\mathrm{P} \ldots \mathrm{P})\end{array}$ & Base soluble \\
\hline SAMPLE 2 & $p$-cresol & & $m$-cresol & $m$-cresol & $\begin{array}{l}\text { "High ortho", alternating } \\
\text { (P-M-P-M...P) }\end{array}$ & Base soluble \\
\hline SAMPLE 3 & $m$-cresol & & $p$-cresol & & Random & Base soluble \\
\hline SAMPLE 4 & Cardanol (C) & & $m$-cresol & $m$-cresol & $\begin{array}{l}\text { "High ortho", alternating } \\
\text { (M-C-M-C...M) }\end{array}$ & Base soluble \\
\hline
\end{tabular}

Table 1. Some of the synthesized novolac copolymers used in this study. 
The 'high ortho' novolac copolymers were prepared by a two-step process (figure 5) (Roy et al 2003d). The first cresolic monomer (designated as ' $I$ ' in table 1) was mixed with formaldehyde $(37 \%)$ and sodium hydroxide in $1: 2 \cdot 2: 1$ ratio. The mixture was stirred mechanically at room temperature $\left(25^{\circ} \mathrm{C}\right)$ for $48 \mathrm{~h}$. The ratio of formaldehyde : cresolic monomer was carefully optimized and the reaction temperature was kept below $25^{\circ} \mathrm{C}$ to avoid oligomer formation in the presence of base. The second cresolic precursor (designated as 'II' in table 1) was then added to the first cresolic monomer in $1: 1$ ratio. The mixture was diluted with hydrochloric acid $(1: 1)$ in an ice bath and the $\mathrm{pH}$ brought down to $4-4 \cdot 5$. The bishydroxymethylated cresol (BHMC) was separated, and the organic layer was washed thoroughly twice with water containing $1 \%$ oxalic acid.

In the second step, the third cresolic monomer (designated as 'III' in table 1) was mixed with liquid BHMC in $1: 1$ ratio with the first phenolic monomer. A $1 \%$ molar equivalent of oxalic acid (with respect to the total cresolic components) was added. Xylene was selected as the reaction medium to produce an azeotropic mixture with water produced during polymerization and the water was tapped out by using the Dean-Stark-trap. The mixture was refluxed at $140-150^{\circ} \mathrm{C}$ for $45 \mathrm{~min}$ and then steam-distilled at $180^{\circ} \mathrm{C}$ for another hour.

The resultant resins were purified by dissolving them in ethyl acetate and reprecipitation was done using $n$-hexane. Finally, novolac resins were dried in vacuo at $30^{\circ} \mathrm{C}$ for $24 \mathrm{~h}$. Molecular weights of the resins were determined by gel permeation chromatography (GPC) using a polystyrene standard column with paraffin as the reference standard and tetrahydrofuran as the eluent.

Photoresists were prepared by mixing appropriate photoactive compounds (diazonaphthoquinone esters for positive and negative photoresists, the aromatic monoazide, 1-azido2, 5-dichloro-3, 6-dimethyl-4-nitro benzene (Eswaran and Sajadian 1988) prepared in our laboratory earlier were used) and novolac resins in the ratio $1: 5$ and then dissolved in ethyl cellosolve acetate. The photoresist solutions were then filtered through $0.45 \mu \mathrm{m}$ teflon filter without applying pressure. Photoresists were then spin-coated on silicon wafers by spin coating at $2000 \mathrm{rpm}$ for $20 \mathrm{~s}$. The prebake was done at $60^{\circ} \mathrm{C}$ for $20 \mathrm{~min}$ in the case of positive photoresists and at $60^{\circ} \mathrm{C}$ for $15 \mathrm{~min}$ in the case of negative photoresists. A 200-watt g-line UV lamp was used for exposure. Developer concentrations were carefully estimated for obtaining the best pattern formation. The patterns obtained were examined by scanning electron microscopy (SEM).

The NMR spectra were recorded at room temperature on a BRUKER SPECTROSPIN DPX-300 MHz spectrometer. The sample solutions were prepared by dissolving $30 \mathrm{mg}$ of sample in $0.5 \mathrm{ml} \mathrm{DMSO}-d_{6}$ for the 1-D spectra and $100 \mathrm{mg}$ of sample in $0.5 \mathrm{ml}$ DMSO- $d_{6}$ for the 2-D NMR spectra. ${ }^{1} \mathrm{H}-\mathrm{NMR}$ spectra were recorded at $300 \cdot 13 \mathrm{MHz}$ whereas 'proton decoupled' ${ }^{13} \mathrm{C}-\mathrm{NMR}$ spectra were recorded at $75.4 \mathrm{MHz}$. TMS was used as internal standard. The pulse delay for inverse gated broadband proton-decoupled quantitative ${ }^{13} \mathrm{C}-\mathrm{NMR}$ spectrum was $10 \mathrm{~s}$ and for TOCSY experiment the mixing time was set at $80 \mathrm{~ms}$.

\section{Results and discussion}

From the series of novolac resins, four resins were selected for preparing photoresists. The first novolac resin had an optimum polydispersivity value $\left(M_{\mathrm{w}}=6251, M_{\mathrm{n}}=815\right.$, $M_{\mathrm{p}}=486, \mathrm{PD}=7 \cdot 6$ ) prepared by the two-step procedure and numbered SAMPLE 1 . The second novolac resin was also synthesized by the two-step procedure; however, it had a low molecular weight and of low PD value $\left(M_{\mathrm{w}}=574\right.$, $\left.M_{\mathrm{n}}=360, M_{\mathrm{p}}=325, \mathrm{PD}=1 \cdot 56\right)$ and numbered SAMPLE 2. The third novolac selected was prepared by the one-step procedure $\left(M_{\mathrm{w}}=9295, M_{\mathrm{n}}=628, M_{\mathrm{p}}=502, \mathrm{PD}=14.8\right)$ and numbered SAMPLE 3. The fourth novolac resin was picked up from the series of CNSL based novolac resins prepared by the two-step procedure $\left(M_{\mathrm{w}}=7394, M_{\mathrm{n}}=6920\right.$, $\left.M_{\mathrm{p}}=502, \mathrm{PD}=1.05\right)$ and numbered SAMPLE 4.

According to the 'stone wall model' (Hanabata et al 1989), the positive photoresist prepared using SAMPLE 1 should show better lithographic performance than SAMPLE 2. We have actually found that in the positive photoresist prepared from SAMPLE 2, the unexposed part of the pattern was severely affected by the developer during development. Sharp edge profiles have been obtained in the case of SAMPLE 1. Presence of the higher molecular weight fraction in the novolac resin thus indeed helps in inhibiting dissolution of the unexposed part of photoresists, as proposed in the 'stone wall model'. The positive photoresists prepared using SAMPLE 3 showed poor lithographic performance. The base solubility of this novolac resin was low and the developer concentration thus required for the photoresist using SAMPLE 3 was thus quite high. The high concentration of base affected the unexposed part of photoresist, with the result that poor edge profiles were observed using SAMPLE 3, as seen in the scanning electron microscopy (figure 4) (Roy et al 2003e).

The negative photoresist prepared using SAMPLE 2 did not show good lithographic performance as the low molecular weight of the novolac resin actually got washed out from the exposed part. SAMPLE 1 did display sharp edges; however, the distinct colour change in the exposed photoresist film during development indicates that base had affected the exposed part (figure 5). Contrary to positive photoresists, the negative photoresist prepared using SAMPLE 3, however, showed patterns with sharp edge profiles. The high stability of photoresist film (novolac resin) in the strong alkaline medium is, therefore, crucial for getting high-resolution geometries in negative photoresist.

The degree of 'high ortho' content and incorporation of different monomers in the novolac resin are the two other 


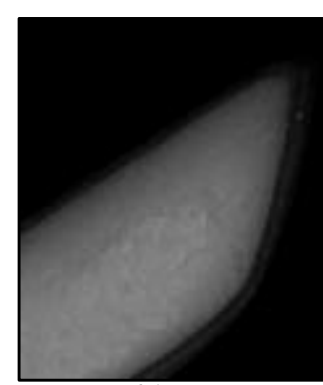

41

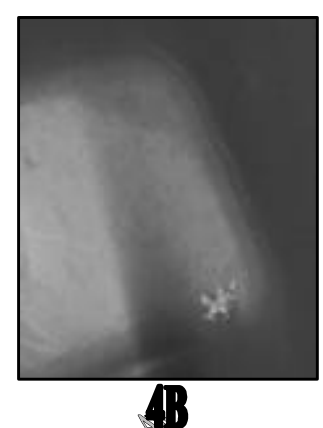

$\mathbb{B}$
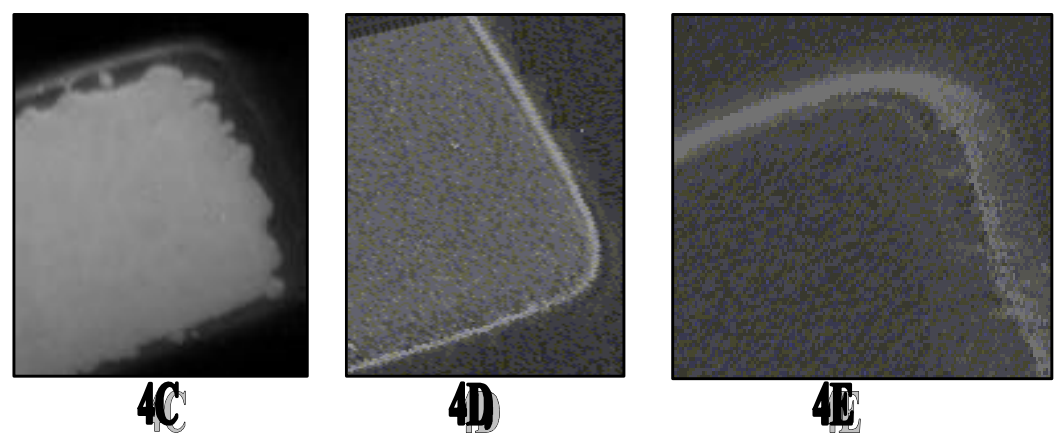

Figure 4. The scanning electron microscopic (SEM) photographs of patterns obtained by photoresists using A. SAMPLE 1, B. SAMPLE 2, C. SAMPLE 3, D. SAMPLE 4 and $\mathbf{E}$ is commercially available photoresist. The sample holder was rotated at $70^{\circ}$ to get the edge profile of the patterns.

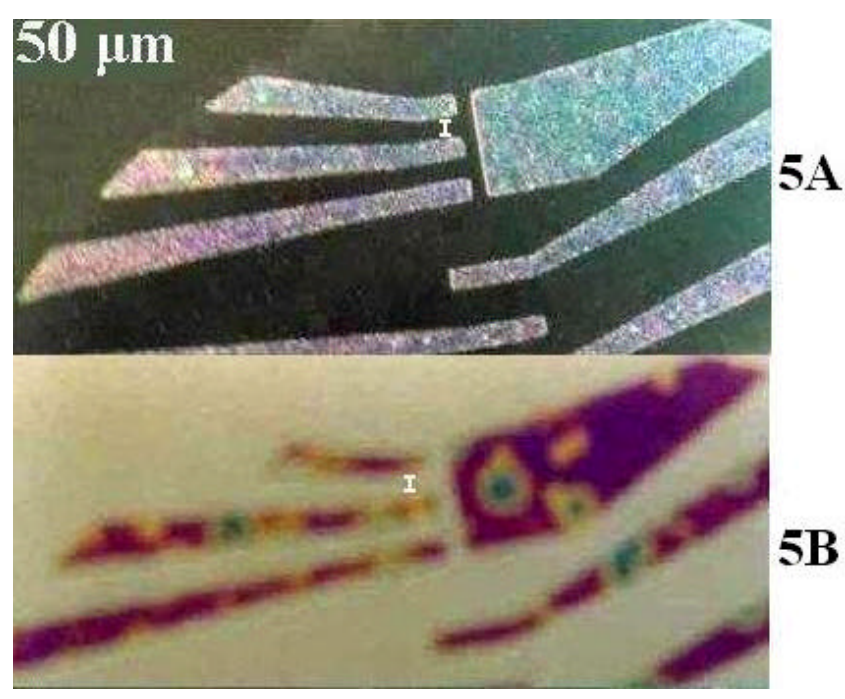

Figure 5. Patterns obtained with negative photoresist using A. SAMPLE 1 and B. SAMPLE 3. The distinct colour change of photoresist film A from green to pink indicates that base has affected the exposed part of photoresist during development.

important criteria for critically determining the lithographic performance of a photoresist. SAMPLES 1, 2 and 3 were examined by 1-D and 2-D NMR spectroscopy for quantitative estimation of linearity and percentage incorporation of different precursors in the polymer skeletons. A modified methodology is proposed here, using the intensities of ${ }^{1} \mathrm{H}$ chemical shifts for assessment of linearity and different monomer percentages in the resin microstructure.

For better lithographic performance, as described above, a range of molecular weights in the novolac microstructure is very crucial. However, their ${ }^{1} \mathrm{H}-\mathrm{NMR}$ spectra becomes quite broad and overlapping (Roy et al 2003e). It is thus difficult to identify the signals for aromatic protons attached to C-5 and C-4 carbon atoms of $m$-cresol individually for $U_{\mathrm{n}}$ value calculation. The additional isomeric possibility in $m$-cresol ('hindered' and 'unhindered') makes the peak assignment really difficult (Bogan and Wolk 1992).

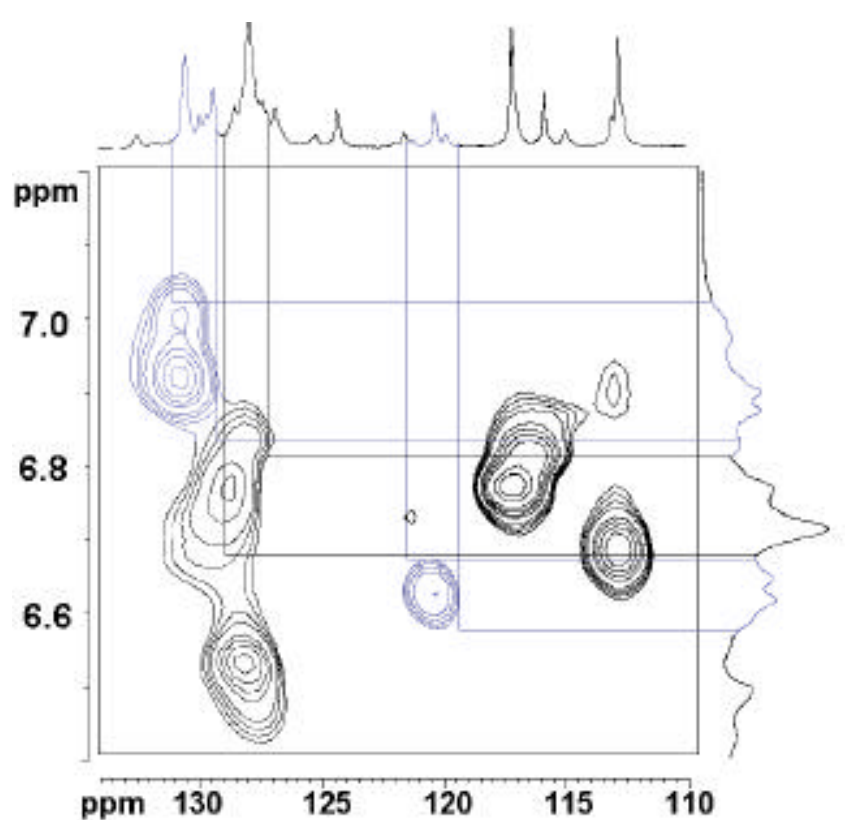

Figure 6. A typical expanded ${ }^{13} \mathrm{C}-{ }^{1} \mathrm{H}$ HETCOR (HSQC) correlation of SAMPLE 2. The gray traces define the C-5 and C-4 and hydrogens attached to the C-5 and C-4, respectively, of $m$ cresol incorporated in the polymer. The black trace around 127$129 \mathrm{ppm}$ is for identical C-5 and C-3 and hydrogens attached to these carbon atoms of $p$-cresol incorporated in the resin.

The 'proton decoupled ${ }^{13} \mathrm{C}-\mathrm{NMR}$ signals are usually sharp and well separated compared to the ${ }^{1} \mathrm{H}-\mathrm{NMR}$ signals. We have, therefore, used the ${ }^{1} \mathrm{H} \rightarrow{ }^{13} \mathrm{C} \rightarrow{ }^{1} \mathrm{H}$ 'round trip' polarization property of the HSQC experiment to deconvolute the aromatic protons in SAMPLE 2 (figure 6). For further confirmation of our ${ }^{1} \mathrm{H}$ chemical shift assignments, we have recorded the DQF-COSY and TOCSY spectra of SAMPLE 2. The expected correlations prove that the above ${ }^{1} \mathrm{H}$ chemical shifts are correct. The $m$-cresol incorporation in SAMPLE 2, as estimated from the deconvoluted ${ }^{1} \mathrm{H}-\mathrm{NMR}$ spectrum appears to be $63.3 \%$ (figure $7 \mathrm{~A}$ ). However, the aromatic region in ${ }^{1} \mathrm{H}-\mathrm{NMR}$ spectrum of SAMPLE 1 was 
highly overlapped, making the deconvolution very difficult (figure 7B). The percentage incorporation of different monomers in the polymer skeleton has therefore been calculated using a different methodology based on the comparison of the area under the curve of methyl protons of $m$-cresol to that of the $p$-cresol (Roy et al 2003e). The percentage $m$-cresol content in the novolac resin is therefore,

$$
\% m \text {-cresol }=\left(A_{m-\mathrm{C}} \times 100\right) /\left(A_{m-\mathrm{C}}+A_{p-\mathrm{C}}\right),
$$

where $A_{m-\mathrm{C}}$ is the area under the curve of the ${ }^{1} \mathrm{H}-\mathrm{NMR}$ peaks for methyl group in $m$-cresol and $A_{p-\mathrm{C}}$ the area under the curve of the ${ }^{1} \mathrm{H}-\mathrm{NMR}$ peaks for methyl group in $p$-cresol.

The identification of $m$ and $p-\mathrm{CH}_{3}$ signals is thus very important. The proton decoupled ${ }^{13} \mathrm{C}$-NMR spectrum of novolac resin has been first investigated, as the ${ }^{13} \mathrm{C}$ chemical shifts are sharper and better separated compared with ${ }^{1} \mathrm{H}$ chemical shifts. The ${ }^{13} \mathrm{C}$-NMR spectrum of SAMPLE 2
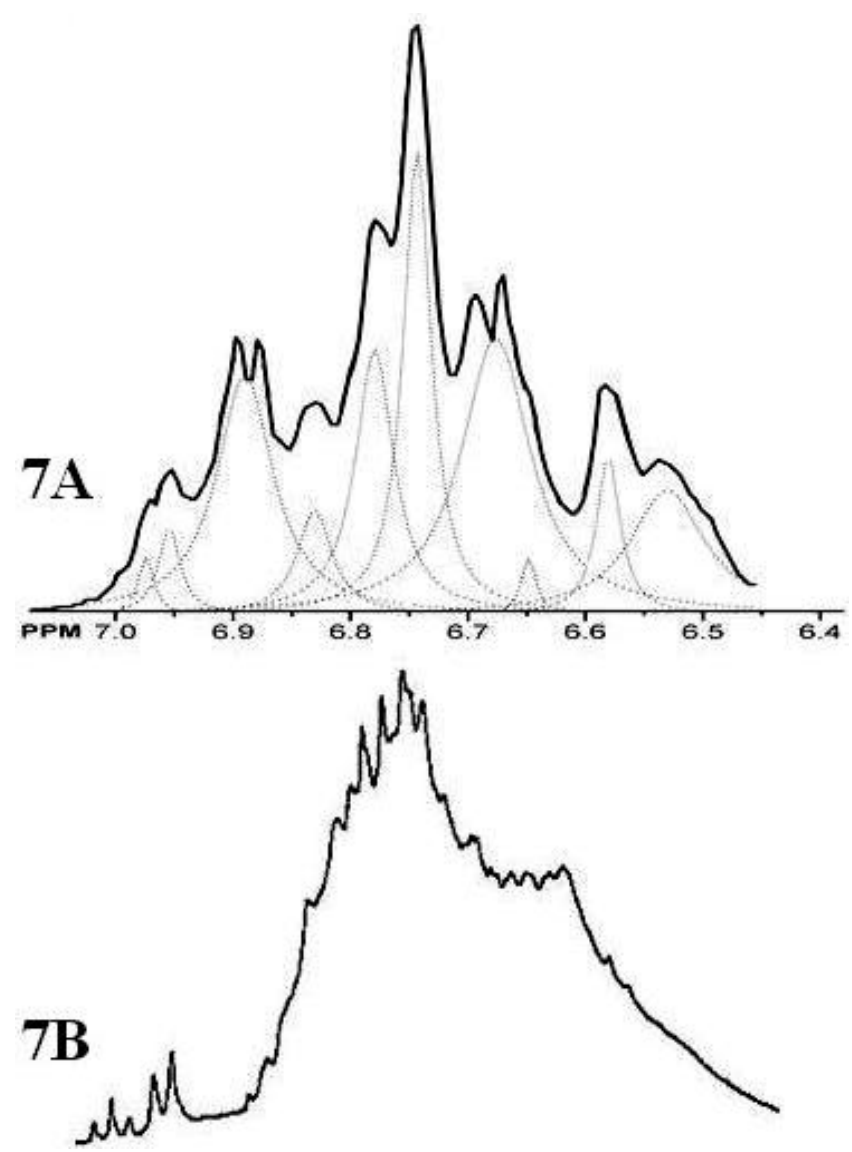

Figure 7. A. The expanded aromatic region of the fully relaxed ${ }^{1} \mathrm{H}-\mathrm{NMR}$ spectrum of SAMPLE 2 . The solid lines indicate the actual ${ }^{1} \mathrm{H}-\mathrm{NMR}$ spectrum. The dotted traces are best-fitting Lorentzian curves. The area under the curve is calculated using a programme written in Qbasic. The aromatic region of the ${ }^{1} \mathrm{H}$ NMR spectrum of SAMPLE 1 is shown in $\mathbf{B}$. The mixture of molecular weights in the novolac morphology (SAMPLE 1) makes the ${ }^{1} \mathrm{H}$ chemical shifts very broad and overlapping, making signal assignment and deconvolution difficult. is compared with the DEPT-135 spectrum for a complete assignment of aliphatic region of the ${ }^{13} \mathrm{C}$-NMR spectrum (figure 8). The peaks at $19 \cdot 2$ and 19.6 are ascribed to the 'hindered' and 'unhindered' $-\mathrm{CH}_{3}$ group of $p$-cresol in the resin; similarly, the signals at 20.6 and $21.0 \mathrm{ppm}$ are assigned to 'hindered' and 'unhindered' $-\mathrm{CH}_{3}$ of $m$-cresol (figure 8) (Mukoyama et al 1973; Roy et al 2003e). Using the HSQC spectrum, the ${ }^{1} \mathrm{H}$ chemical shifts corresponding to ${ }^{13} \mathrm{C}$ chemical shifts were then identified. The ${ }^{13} \mathrm{C}$ chemical shifts at 19.2 and $19.6 \mathrm{ppm}$ are correlated well with the ${ }^{1} \mathrm{H}$ chemical shift at $\delta=2 \cdot 1 \mathrm{ppm}$, thereby identifying the ${ }^{1} \mathrm{H}$ chemical shift of methyl group of $p$-cresol to be at $\delta=2 \cdot 1 \mathrm{ppm}$. Likewise, the signals at 20.6 and $21.0 \mathrm{ppm}$ of $m$-cresol in ${ }^{13} \mathrm{C}$-NMR spectrum are seen to be correlated with the signal at $\delta=2 \cdot 2 \mathrm{ppm}$ in ${ }^{1} \mathrm{H}-\mathrm{NMR}$ spectrum. For a decisive confirmation of the ${ }^{1} \mathrm{H}$ chemical shift assignments, the TOCSY experiment was carried out, using a longer mixing time of $80 \mathrm{~ms}$ to allow magnetization transfer to remote protons, thus enabling the long range correlation between methyl proton and aromatic hydrogens to be seen in the TOCSY spectrum. The protons of methylene bridges also show an expected correlation with methyl protons. The ${ }^{1} \mathrm{H}$ chemical shift assignments for methyl protons of $p$-cresol at $\delta=2 \cdot 1 \mathrm{ppm}$ and of $m$-cresol at $\delta=$ $2 \cdot 2 \mathrm{ppm}$ are therefore confirmed (Roy et al 2003e).

The result based on aliphatic hydrogens is in quite good agreement with that based on aromatic protons in the case of SAMPLE 2. The $m$-cresol content in SAMPLE 2 is $60 \cdot 1 \%$ using methyl protons for comparison, while a comparison of the aromatic hydrogen resonances led to $63.3 \%$. Comparing methyl proton intensities from the deconvoluted ${ }^{1} \mathrm{H}-\mathrm{NMR}$ spectra, the percentage incorporation of $m$-cresol in SAMPLES 1 and 2 are calculated as $66.25 \%$ and $31.97 \%$, respectively (Roy et al 2003e).

Calculation of percentage incorporation of cardanol in the cardanol based novolac polymer, however, necessitates modification of the above methodology. Cardanol has a structure very similar to that of $m$-cresol, the only difference being that cardanol has a long side chain of $-\mathrm{C}_{15} \mathrm{H}_{25-31}$ in place of the methyl group in $m$-cresol (figure 9). The percentage incorporation of cardanol in SAMPLE 4 will thus be the ratio of area under the curve of protons for $-\mathrm{CH}_{3}$ of side chain $\left(-\mathrm{C}_{15} \mathrm{H}_{25-31}\right)$ to that of $-\mathrm{CH}_{3}$ of $m$-cresol. It has been well known that the cardanol is a mixture of four compounds having different types of side chains containing both the methyl group and double bonded methylenic group as the end group (Tyman 1979). Using DEPT-135, HSQC, DQF-COSY and TOCSY experiments, we have shown that the signal at $\delta=4.9 \mathrm{ppm}$ is due to the terminal methylene group of cardanol side chain (figure 10) (Roy et al 2003d). Therefore, for the quantitative estimation of cardanol in SAMPLE 4, the formula has been revised to

$$
\% m \text {-cresol }=\left(A_{m-\mathrm{C}}\right) /\left(A_{m-\mathrm{D}}+A_{e-\mathrm{D}}\right),
$$

where $A_{m-\mathrm{C}}=$ area of signals for $-\mathrm{CH}_{3}$ of $m$-cresol in proton NMR spectrum, $A_{m-\mathrm{D}}=$ area of signals for $-\mathrm{CH}_{3}$ 


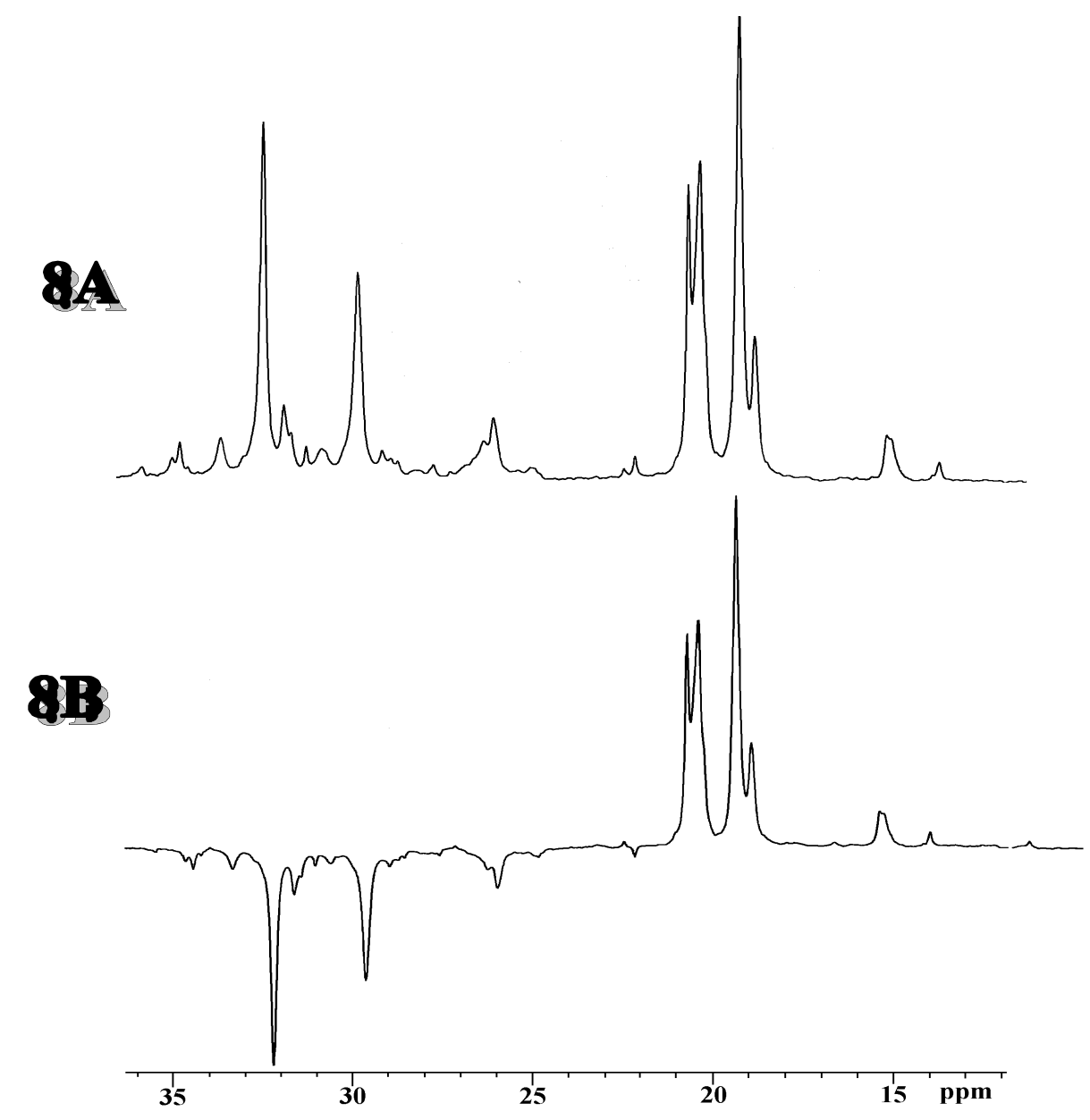

Figure 8. Comparison of the aliphatic region in the spectra of SAMPLE 2. In A, the ${ }^{13} \mathrm{C}-\mathrm{NMR}$ spectrum is shown whereas the DEPT-135 spectrum is presented in $\mathbf{B}$. The two pairs of peaks at 19.2 and 19.6 and 20.6 and $21.0 \mathrm{ppm}$ indicate that these represent the methyl carbon atoms.

and $A_{e-\mathrm{D}}=$ area of signals for $-\mathrm{CH}_{2}$ of cardanol side chain.

The percentage of $m$-cresol incorporation in SAMPLE 4 was calculated to be $50 \cdot 34 \%$ from fully relaxed deconvoluted ${ }^{1} \mathrm{H}$-NMR (figure 11 ).

The higher incorporation of $m$-cresol in the novolac copolymer thus increases the base solubility of the resin. The novolac copolymer with an optimum percentage of $m$-cresol and $p$-cresol (around $60: 40$ ) is thus useful for positive photoresist formulation, as we need the higher alkaline solubility of the novolac resin for a good pattern formation. SAMPLE 1 has $66.25 \% \mathrm{~m}$-cresol, and is therefore more soluble in base compared to SAMPLE 3 and consequently shows a better lithographic performance as a positive photoresist. The novolac resin with $68.03 \% p$ cresol (SAMPLE 3) is strongly base-resistant and performed well in lithographic experiment using negative photoresist (Roy et al 2003e).

The exact determination of the degree of linearity (represented by $U_{4}$ ) also required a thorough retrospection of the calculation methodology. The ${ }^{1} \mathrm{H}-\mathrm{NMR}$ spectrum of high

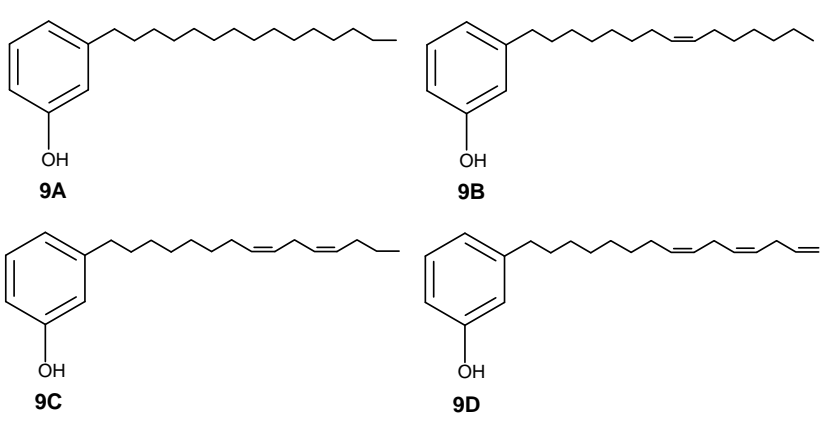

Figure 9. Natural cardanol is a mixture of four compounds having different types of side chains: In figures $\mathbf{A}, \mathbf{B}, \mathbf{C}$, the terminal group is methyl, and in $\mathbf{D}$ a double bonded methylenic group is the end group.

molecular weights polymer with a range of molecular weight, particularly the aromatic region is often very broad and overlapping with weak shoulders, rendering deconvolution difficult. Calculation from the comparison of 
methyl region is not effective as the linearity involves the substitution/unsubstitution of the aromatic moiety. We therefore recorded an inverse gated broadband protondecoupled quantitative ${ }^{13} \mathrm{C}-\mathrm{NMR}$ spectrum for the estimation of $U_{4}$ (figure 12) (Roy et al 2003e). The signals at $121.6-125.6 \mathrm{ppm}$ in the inverse gated broadband protondecoupled quantitative ${ }^{13} \mathrm{C}-\mathrm{NMR}$ spectrum are assigned to the C-4 of $m$-cresol and the signals at $129 \cdot 8-130 \cdot 3 \mathrm{ppm}$ are assigned to the $\mathrm{C}-5$ of $m$-cresol incorporated in the resin (Bogan and Wolk 1992; Roy et al 2004b). $U_{4}$ value in this case will therefore be defined as,

$$
U_{4}=\left(A_{4} \times 100\right) /\left(A_{5} \times I_{\mathrm{n}}\right)
$$

where $A_{4}$ represents the area under the curve for C-4 of $m$-cresol incorporated in the resin, $A_{5}$ represents the area under the curve for $\mathrm{C}-5$ of $m$-cresol incorporated in the resin and $I_{\mathrm{n}}$ the intensity correlation factor which is considered as 1 .

The $U_{4}$ calculated for SAMPLE 2 using the quantitative ${ }^{13} \mathrm{C}$-NMR method is $57 \cdot 4$, which is very well corrobo-

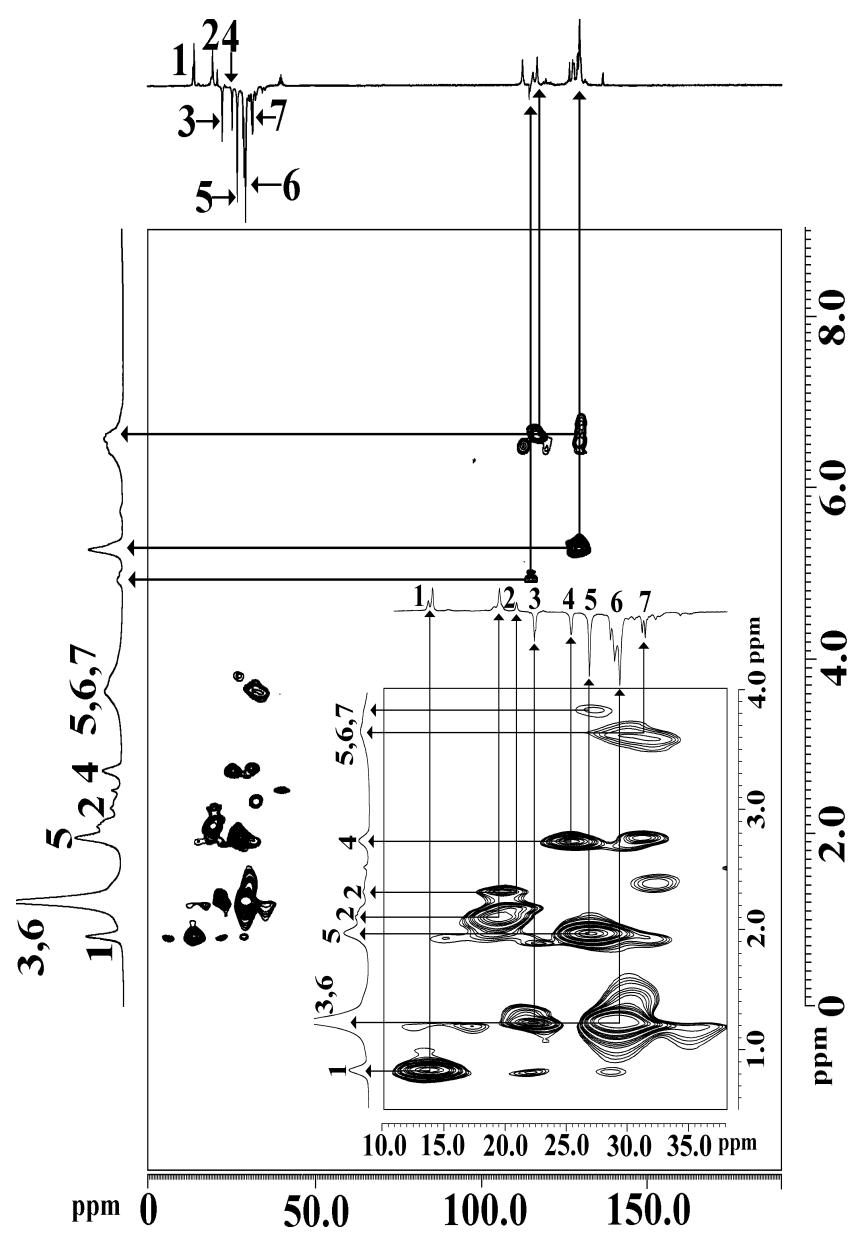

Figure 10. HSQC spectrum of SAMPLE 4. The DEPT-135 spectrum is plotted along $\mathrm{x}$-axis and the ${ }^{1} \mathrm{H}-\mathrm{NMR}$ spectrum is plotted along y-axis. In the inset, the expanded aliphatic region correlations are shown for clarity. rated by the $U_{4}$ value (58.9) calculated based on ${ }^{1} \mathrm{H}-\mathrm{NMR}$ chemical shift. The $U_{4}$ values of SAMPLES 1 and 3 were estimated to be $56.02 \%$ and $60.58 \%$, respectively. Most surprisingly, the novolac resin prepared by our one-step method (SAMPLE 3) showed the highest $U_{4}$ value compared to those synthesized based on two-step procedure. SAMPLE 3 has very high $p$-cresol incorporation, and since the $p$-cresol has only two ortho positions free for polymerization, the greater $p$-cresol incorporation will increase the linearity ("high ortho" nature) in the resin microstructure. The novolac resin prepared by our one-step procedure was leached out with alkali, whereby the more ordered

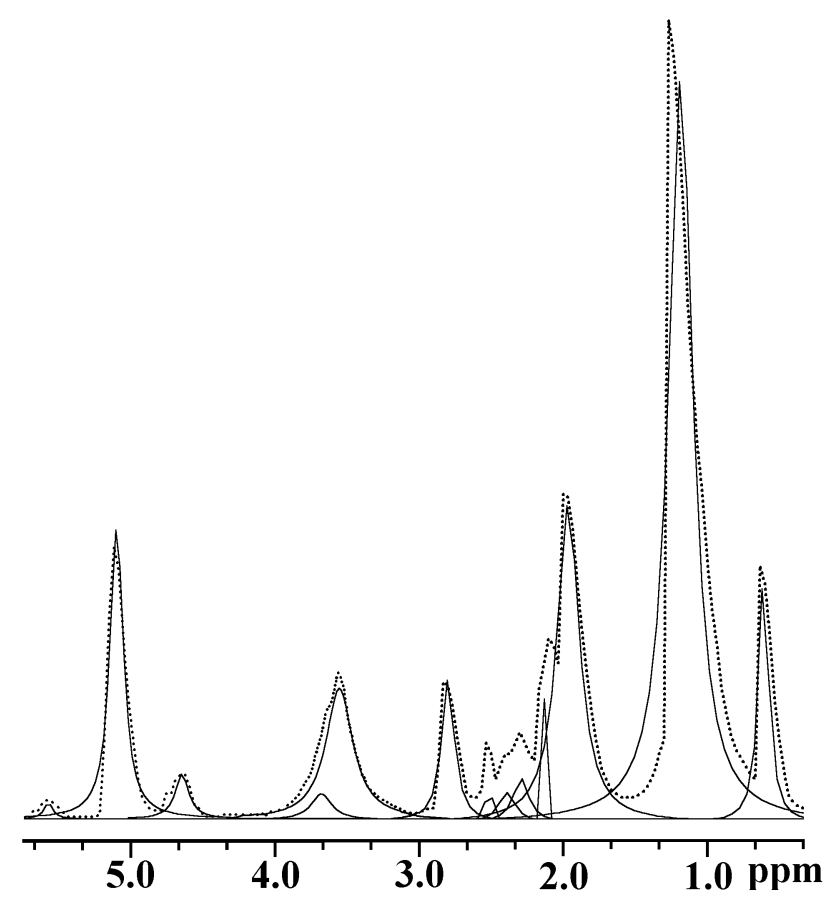

Figure 11. Deconvoluted ${ }^{1} \mathrm{H}-\mathrm{NMR}$ spectrum of SAMPLE 4. The dotted line corresponds to the original ${ }^{1} \mathrm{H}-\mathrm{NMR}$ spectrum, while the solid traces are best-fitting Lorentzian curves.

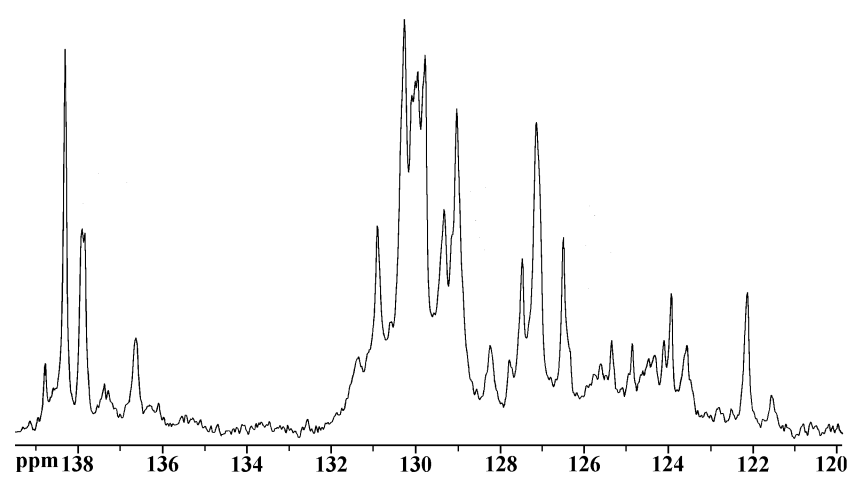

Figure 12. The inverse gated broadband proton-decoupled quantitative ${ }^{13} \mathrm{C}-\mathrm{NMR}$ spectrum of SAMPLE 2 . The pulse delay was set at $10 \mathrm{~s}$ to get a fully relaxed spectrum. 
<smiles>CCCc1c(C)ccc(Cc2cc(C)cc(Cc3ccc(C)c(CCC)c3O)c2O)c1O</smiles><smiles>N=C1C=Cc2c(cccc2S(=O)(=O)Oc2c(C(=O)c3ccccc3)ccc([N+](=O)[O-])c2O[N+](=O)[O-])C1=O</smiles>

13B<smiles></smiles>

Figure 13. Hanabata's proposed mechanism for the unexposed positive photoresist: A. the original novolac resin, B. the diazonaphthoquinone-5-sulphonic acid esterified with 2,3,4-trihydroxybenzophenone and $\mathbf{C}$. the unexposed photoresist based on $\mathbf{A}$ and $\mathbf{B}$ after development with base. polymer chains with higher $p$-cresol content went into basic solution, and the polymer was reprecipitated using dilute $\mathrm{HCl}$. The alkali leaching technique therefore could be successfully used to fractionate the more ordered polymer from the random one. Ordered novolac copolymers with an optimum $m$ and $p$-cresol content and optimum base solubility and giving microlithographic patterns with sharp edge profiles, should prove useful for the design and preparation of high-performance photoresists (Roy et al 2003e).

A clear insight into the mechanism of action of photoresists is also very critical for the design of high performing photoresists. It has been suggested that, in the unexposed regions, DNQ undergoes an azo coupling reaction at the 'free para' position of $m$-cresol incorporated in the novolac polymer (figure 13) (Hanabata et al 1989; Ueno 1998). Linear 'high ortho' novolacs, with a higher free para position content, show superior lithographic performance compared with the random resins.

Based on molecular modelling, ${ }^{1} \mathrm{H}-\mathrm{NMR},{ }^{13} \mathrm{C}-\mathrm{NMR}$ and DEPT-135 spectral studies, we have recently shown that in the unexposed positive photoresist on development with base, the diazonaphthoquinone and the novolac resin undergo an azo coupling reaction at the methylene bridges rather than at the para position of the novolac resin. In our molecular modelling study, the azo-coupled product of monoester of DNQ-5 sulfonic acid with 2,3,4-trihydroxybenzophenone and the methylene hydrogens of novolac resin surprisingly showed a much lower minimum potential energy than that of Hanabata model as shown in figure 14. Sharp differences have been found in the methylenic region at $\delta=$

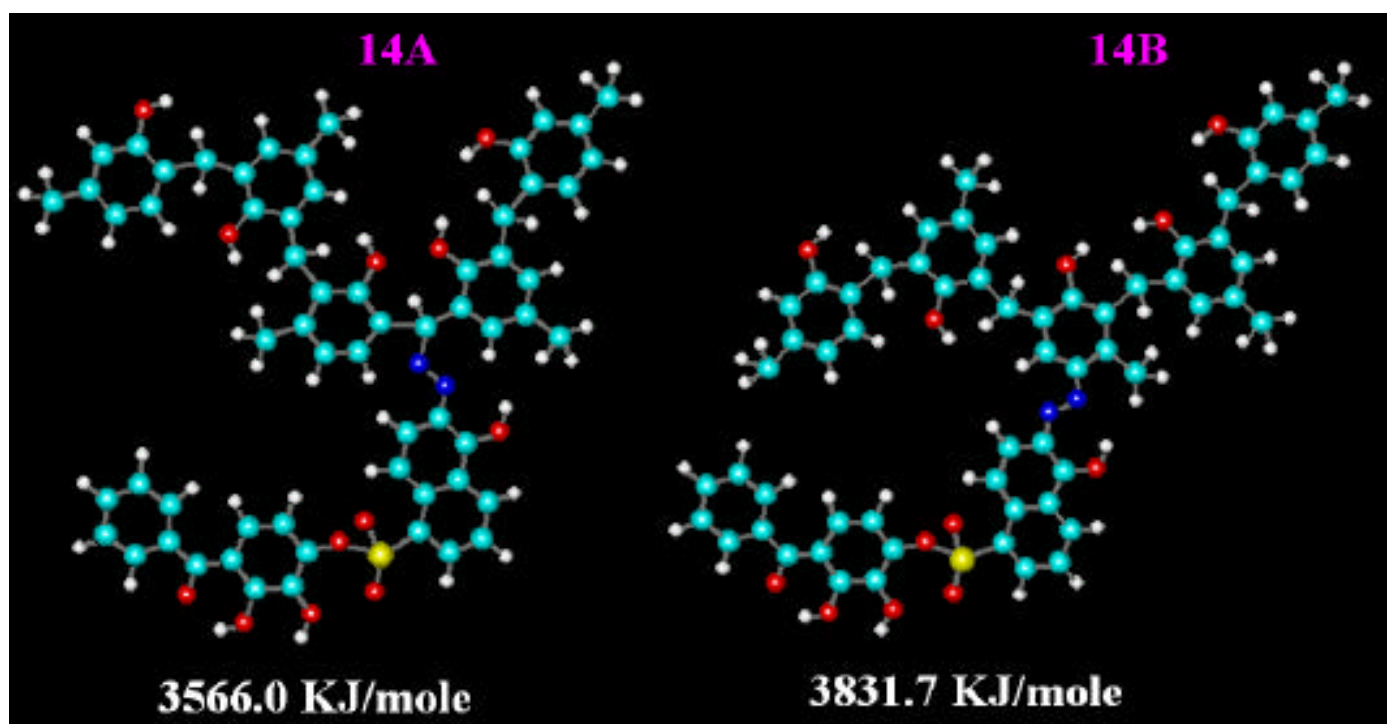

Figure 14. The energy-minimized structures: A. DNQ azo-coupled with methylene bridges of a novolac resin and B. the DNQ azo-coupled with free para positions of $m$-cresol, incorporated in the novolac resin. The energy minimized structures are obtained by geometrical optimization of the structures using molecular mechanics force field; $\mathrm{Mm}+$ and algorithm; Steepest descent method using HyperChem Professional 6.03 molecular modelling software. The cyan colours represent carbon atoms whereas white, red, blue and yellow colours represent hydrogen, oxygen, nitrogen and sulphur, respectively. The potential energy of $\mathbf{A}$ is much lower than that of $\mathbf{B}$ indicating the ease of attack of DNQ on the methylene bridges rather than the para positions of $m$-cresol. 


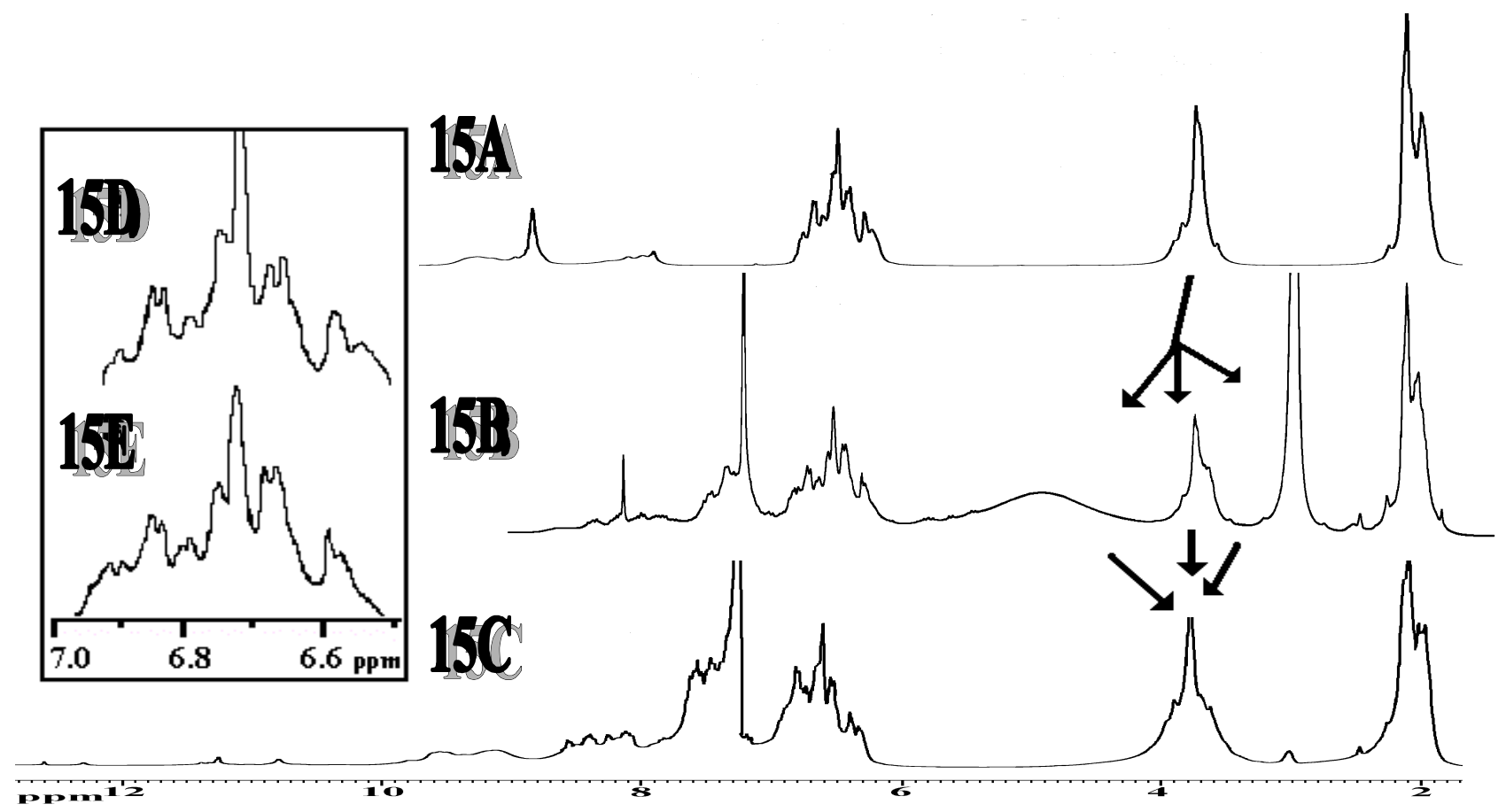

Figure 15. ${ }^{1}$ H-NMR spectrum of the novolac oligomer (A), unexposed (B) and exposed positive photoresists $(\mathbf{C})$. In the inset, the expanded aromatic region of the original novolac oligomer (SAMPLE 2), (D) and unexposed photoresist (E), are shown.

3-5 ppm between the exposed and unexposed parts of photoresist prepared using SAMPLE 2, indicating the attack of DNQ on the methylenic bridge. However, there is no change in the aromatic region in the spectra of the novolac oligomer and unexposed photoresist, as shown in the inset of figure 15 (Roy et al 2003b). On undergoing the azo coupling reaction, the methylene group of the novolac resin would be converted to a methine $(\mathrm{CH})$ group.

To further confirm this fact, we recorded the ${ }^{13} \mathrm{C}-\mathrm{NMR}$ and DEPT-135 spectrum of SAMPLE 2 and photoresist prepared using SAMPLE 2 . The ${ }^{13} \mathrm{C}$ chemical shifts at $54 \cdot 5$ and $79.2 \mathrm{ppm}$ are assigned to the methine carbons coming from the methylenic carbons which have taken part in the azo coupling reaction of DNQ in the case of the unexposed photoresist. These two signals in the case of the unexposed photoresist are 'up' in the DEPT-135 experiment, whereas the other methylene carbons at 26-32 ppm are expectedly 'down' in this DEPT pulse sequence (figure 16) (Roy et al 2003b).

The need for "high ortho" novolacs for better performance of photoresists has been explained due to the linear nature of the polymer. DNQ can attack the methylene bridge, only if it can approach it closely enough (this being a solid state reaction). In crosslinked novolac resins, DNQ will not be able to approach the methylene bridge sufficiently closely (figure 17). The suggested new mechanism successfully explains pattern formation obtained even with photoresists based on $p$-cresol, which do not have any free para positions at all.

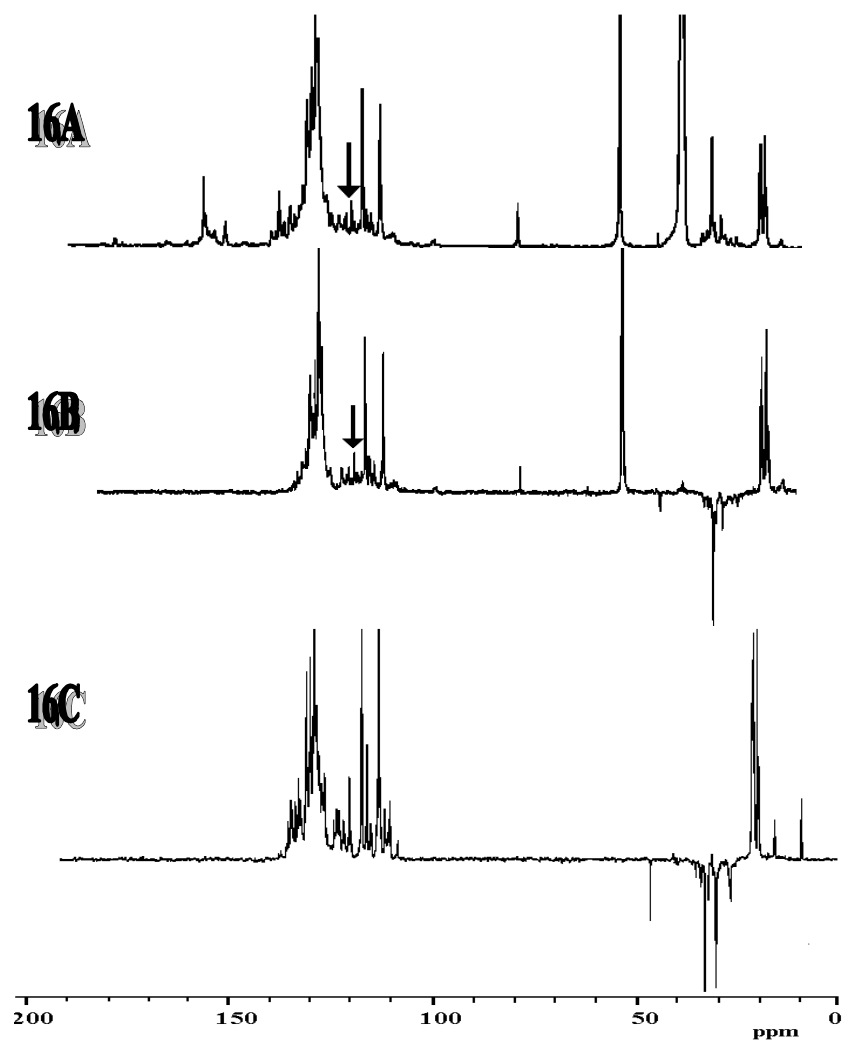

Figure 16. A. The ${ }^{13} \mathrm{C}-\mathrm{NMR}$ spectrum of unexposed photoresist, B. the DEPT-135 spectrum of the same and C. the DEPT-135 spectrum of exposed photoresist. 
Exploiting this change of physical property on exposure of a negative photoresist, a pattern can be transferred to a substrate from a mask. A quinone-imine type complex has been proposed earlier involving the aromatic moiety of novolac resin as the reaction site (figure 18).

A more recent study focuses the attack of nitrene on methylenic bridge and hydroxyl group of novolac resins which were found to be responsible for the crosslinking reaction along with the aromatic moiety of novolac resin (Voigt 1999). This should have led to the presence of signals due to methine hydrogens. However, no such evidence was shown in that study. It was concluded that the collective effects of attacking the methylenic bridge, aromatic moiety and hydroxyl group by nitrene resulted in the increase of molecular weight of resin in the exposed negative photoresist.

The ${ }^{1} \mathrm{H}$-NMR spectra of photoresists prepared using SAMPLE 2, before and after photolysis, do not indicate the involvement of methylene region in the crosslinking reaction, and the same is true for the aromatic moiety. The intensities of the hydroxyl groups become diminished drastically in the proton NMR spectrum of exposed photoresist compared to its unexposed counterpart (figure 19). The fast exchange of hydroxyl protons, however, could make these intensities somewhat unreliable.<smiles>[CH2+]CCc1c(C)ccc(Cc2cc(C)cc(Cc3ccc(C)c(CCC)c3O)c2O)c1O</smiles><smiles>CCCCc1c(C)ccc(Cc2cc(C)cc(C(N=Nc3ccc4c([O-])cccc4c3O)c3ccc(C)c(CCCC)c3O)c2O)c1O</smiles>

Figure 17. Proposed new mechanism: $\mathbf{A}$ is a $m$-cresol $/ p$-cresol based "high ortho" novolac resin; B is 2,1-diazonaphthoquinone5-sulphonic acid esterified with 2,3,4-trihydroxybenzophenone and $\mathbf{C}$ is the unexposed photoresist. DNQ thus attacks the methylene bridges, rather than the aromatic ring as suggested in the earlier mechanism.
To confirm the crosslinking site further, we recorded the ${ }^{13} \mathrm{C}-\mathrm{NMR}$ and DEPT-135 spectra of the negative photoresists. No change in the ${ }^{13} \mathrm{C}$ chemical shifts of methylenic carbon atoms was found between the DEPT-135 spectra of novolac resin and the exposed resist. The aromatic ring of photoactive azide is fully substituted and thus does not show any signal in the aromatic region of DEPT-135 spectrum of the negative photoresist. The almost super-

\section{$18 \mathrm{~A}$}

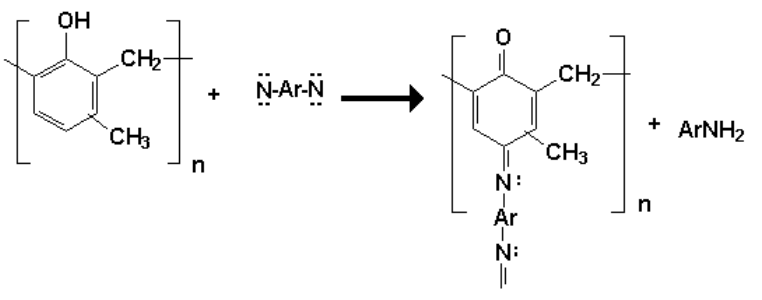

$18 B$

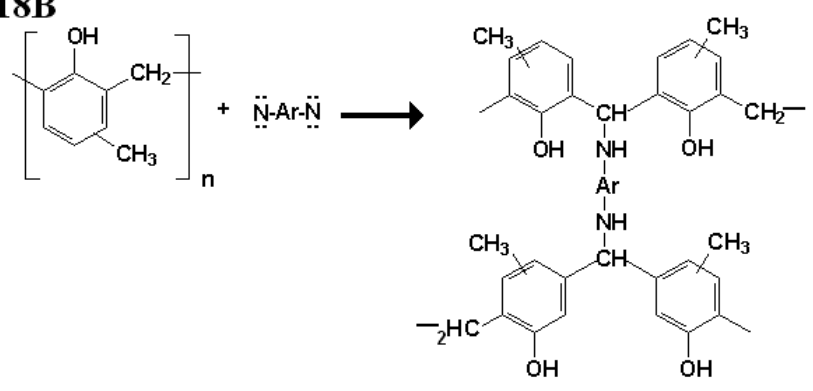

Figure 18. The earlier proposed quinone-imine type of complexation is shown in $\mathbf{A}$ involving aromatic moiety and aromatic bisazide; and $\mathbf{B}$ envisages the attack of nitrene with the methylene bridges of the novolac resin.

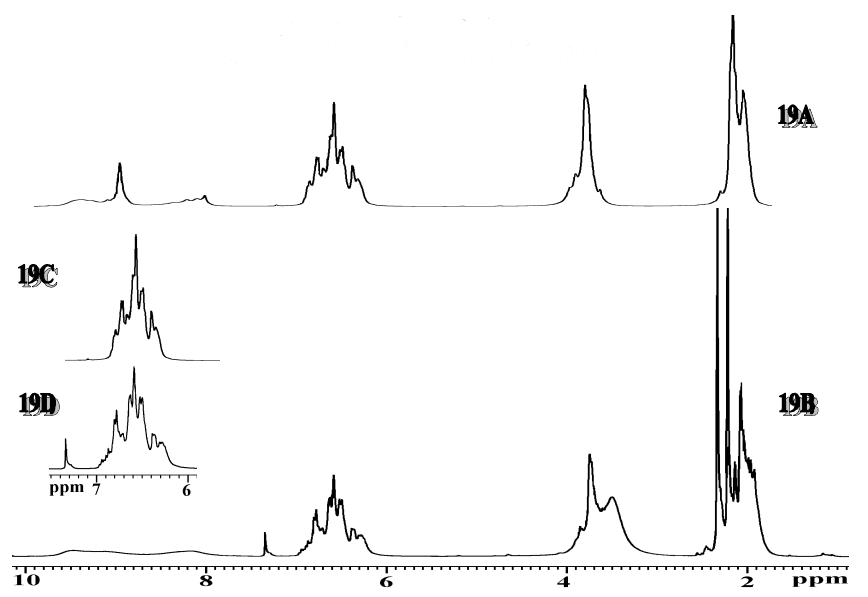

Figure 19. ${ }^{1} \mathrm{H}-\mathrm{NMR}$ spectra of novolac resin (SAMPLE 2) (A) and novolac resin with the aromatic azide after $20 \mathrm{~min}$ of exposure (B). An extra peak at $\delta=3.5 \mathrm{ppm}$ in $\mathbf{B}$ could be due to the moisture coming from hygroscopic DMSO- $d_{6}$ (after exposure, the photoresist became quite insoluble in DMSO- $d_{6}$ and thus required longer time to dissolve the sample). In the inset, the aromatic region is shown, $\mathbf{C}$ and $\mathbf{D}$ represent the novolac resin (SAMPLE 2) and the novolac resin with the aromatic azide after $20 \mathrm{~min}$ of exposure, respectively. 

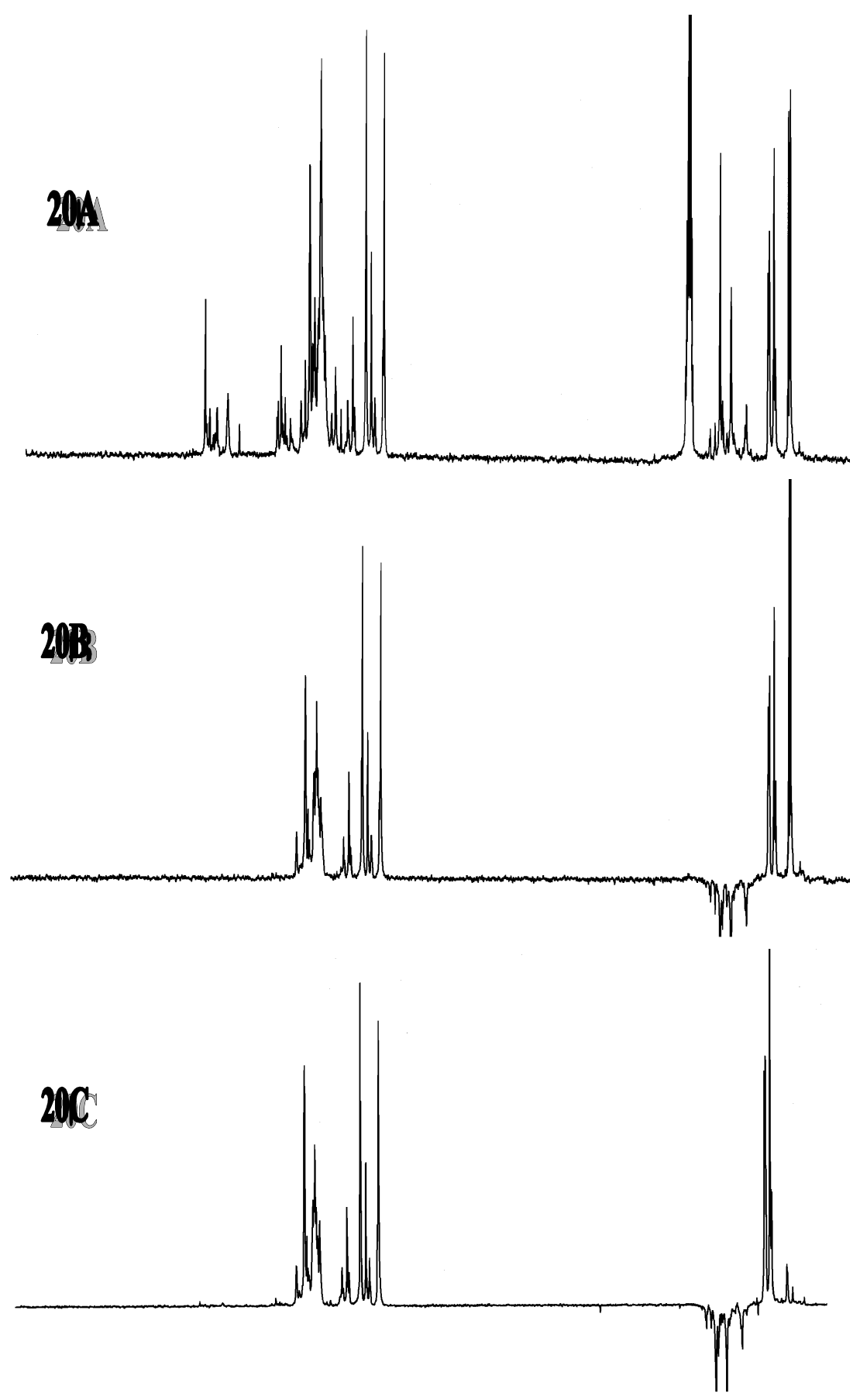

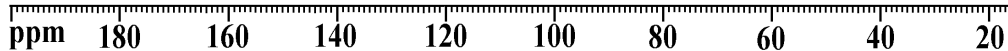

Figure 20. A-B. The ${ }^{13} \mathrm{C}-\mathrm{NMR}$ and DEPT-135 spectra, respectively, of the novolac resin (SAMPLE 2) mixed with azide after $20 \mathrm{~min}$ of exposure and C. the DEPT-135 spectrum of the novolac resin (SAMPLE 2) alone.

imposable ${ }^{13} \mathrm{C}$ signals in the aromatic region of the novolac resin and exposed photoresist conclusively prove the lack of involvement of the aromatic moiety of novolac resin in the crosslinking reaction (figure 20) (Roy et al 2003c).

To obtain a better insight into crosslinking mechanism, we performed a molecular modelling calculation using
HyperChem Professional 6.03 software version. The calculation suggests that the most facile site of attack is the phenolic hydroxyl group (figure 21).

Negative photoresists based on $p$-chlorophenol and monoazide produce equally good patterns as that from $m$-cresol based novolac resin. The attack of nitrene on aromatic 


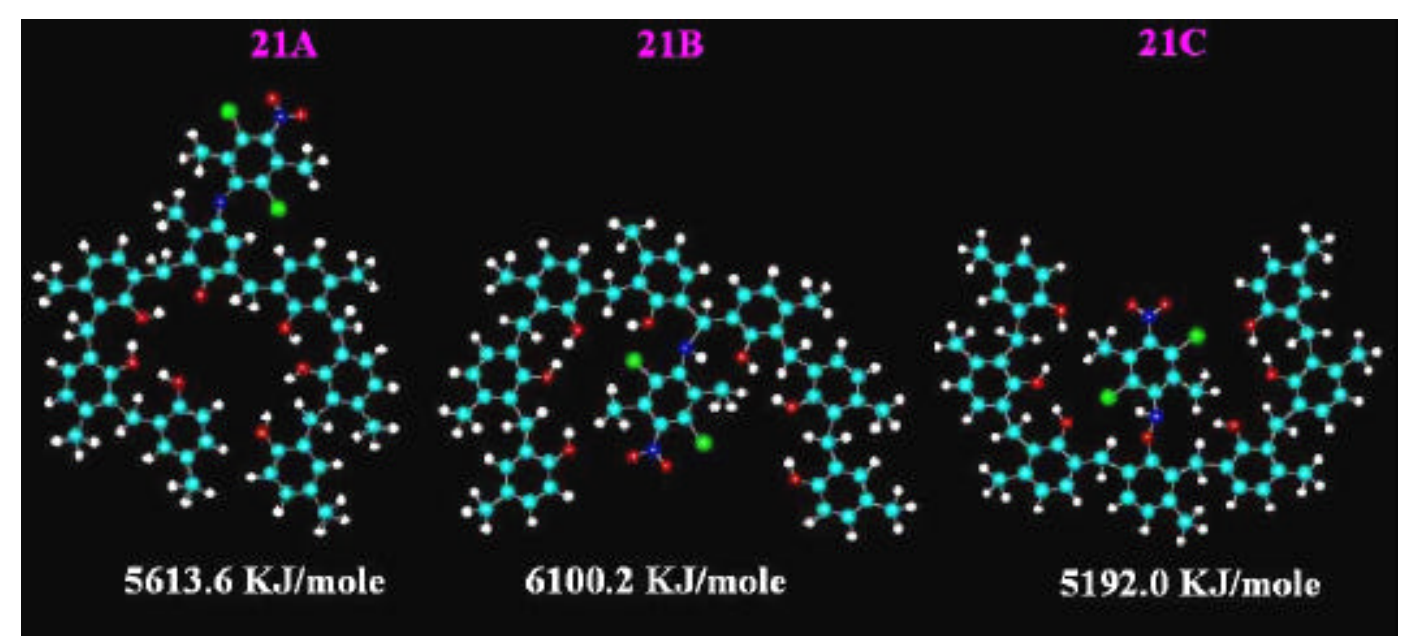

Figure 21. The energy-minimized structures: A-C represent the attack on the aromatic moiety, the methylenic bridge and the hydroxyl group of the novolac resin, respectively. These energy minimized structures are obtained by geometrical optimization of the structures using molecular mechanics force field; $\mathrm{Mm}+$ and the steepest descent algorithm. The cyan colours represent carbon atoms whereas white, red, blue and green colours represent hydrogen, oxygen, nitrogen and chlorine, respectively.<smiles>CCCc1cccc(CC)c1ONc1c(C)c(Cl)c([N+](=O)[O-])c(C)c1Cl</smiles>

Figure 22. The proposed crosslinking mechanism of aryl nitrene with hydroxyl group of novolac resin.

moiety on exposure is ruled out based on the fact that $p$ chlorophenol does not have any free para position in the aromatic ring. The phenolic groups are responsible for the base solubility of novolac resin, and any attack on this functional group should therefore lead to increased insolubility in base. We used a monoazide in place of bisazide, for the negative photoresist preparation, and therefore, no crosslinked novolac is expected. The exposed area of the photoresist becomes insoluble in the alkaline developer not only due to an increase in the molecular weight of the photoresist but also due to a change in the nature of the phenolic functionality which explains the huge polarity difference between the exposed and unexposed parts leading to the dissolution promotion in the unexposed part (figure 22) (Roy et al 2003c).

\section{Conclusions}

Recent years have seen the identification of several optimized parameters for obtaining lithographically better performing photoresists. In the case of the extensively used novolac resins, their microstructure, molecular weights and their distribution, the ordered microstructure and optimum incorporation of the monomers in copolymeric structures have been shown to cause marked solubility differences between exposed and unexposed parts of photoresists. From the chemical synthetic viewpoint, the exact elucidation of microstructure and bonding patterns therefore becomes necessary. The use of a battery of modern NMR techniques has been demonstrated here for obtaining unambiguous molecular level structural information. These microstructural data are shown to have a critical impact on the lithographic performance of photoresists. Using improved 1-D and 2-D NMR techniques conversion of functional group has been quantitatively calculated, and this dramatically affects the physical and chemical properties of the polymer morphology. An assessment of the mechanism of action of photoresists has been made, which in turn helps to design better performing photoresists.

\section{Acknowledgements}

We are grateful to Director, Solid State Physics Lab, Delhi, for the sanction of a project and a fellowship. One of the authors (SVE) thanks UGC for approving a major research project. We thank Dr Vishnu Gopal, Dr Vikram Dhar, Dr Rawal and Ms Asha Gandhi, Solid State Physics Lab, for kind permission to carry out the lithographic experiments, assistance in simulation work, recording scanning elec- 
tron microscopic photographs and for generous help in the lithographic experiments, respectively. Authors also thank Director, IICT, Hyderabad, and Mr Ishwar Singh for supplying DNQ and CNSL/cardanol. We are indebted to Dr Raja Roy, Central Drug Research Laboratory, Lucknow, for recording the inverse gated broadband proton-decoupled quantitative ${ }^{13} \mathrm{C}$-NMR spectra. We are grateful to Prof. Anil Kumar, Sophisticated Instrument Facility, Indian Institute of Science, Bangalore, for very helpful discussions and suggestions. Finally, we would like to extend our sincere gratitude to Prof. Vikram Kumar, Director, National Physical Laboratory, Delhi (ex-Director, Solid State Physics Lab), without his constant encouragement and spontaneous help this piece of work would not have been possible.

\section{References}

Bähr G, Westerwelle U and Gruetzner G 1997 Proc SPIE-Int. Soc. Opt. Eng. 3049628

Blevins R W, Daly R C and Turner S R 1987 Encyclopedia of polymer science and engineering (ed.) J I Krocehwitz (USA: John Wiley \& Sons) 9 p. 107

Bogan L E and Wolk S K 1992 Macromolecules 25161

Eswaran S V, Basu P K and Roy Debmalya 2001 Patent applied for

Eswaran S V British patent, UK 277897 1996; Australian patent, AU 6722201997

Eswaran S V and Sajadian S K 1988 Synth. Commun. 181807

Fitzgerald E A 1990 J. Appl. Polym. Sci. 411809

Hanabata M, Uetani Y and Furuta A 1989 J. Vac. Sci. Technol. B7 640

Hattori K T, Hattori T, Uchino S, Ueno T, Hayashi N, Shirai S, Moriuchi N and Morita M 1992 Jpn. J. Appl. Phys. 314307
Khadim M A, Rahman M D and Durham D L 1992 Proc. SPIEInt. Soc. Opt. Eng. 1672347

Kopf P W 1988 Encyclopedia of polymer science and engineering (ed.) J I Krosehwitz (USA: John Wiley \& Sons) 11 p. 50

Mukoyama Y, Tanno T, Yokokawa H and Fleming J $1973 \mathrm{~J}$. Polym. Sci. 33193

Reiser A, Shih H Y, Yeh T F and Huang J P 1996 Angew. Chem. Int. Ed. Engl. 352428

Roy Debmalya, Basu P K and Eswaran S V 2002 Resonance 7 44, 59

Roy Debmalya, Basu P K, Raghunathan P and Eswaran S V 2003a Polym. Int. 52757

Roy Debmalya, Basu P K, Raghunathan P and Eswaran S V 2003b Magn. Reson. Chem. 4184

Roy Debmalya, Basu P K, Raghunathan P and Eswaran S V 2003c Magn. Reson. Chem. 41671

Roy Debmalya, Basu P K, Raghunathan P and Eswaran S V 2003d J. Appl. Polym. Sci. 891959

Roy Debmalya, Gandhi A, Basu P K, Raghunathan P and Eswaran S V 2003e Microelectron. Eng. 7058

Roy Debmalya, Basu P K, Raghunathan P and Eswaran S V 2004a Magn. Reson. Chem. 4276

Roy Debmalya, Basu P K, Raghunathan P and Eswaran S V 2004b J. Appl. Polym. Sci. 912096

Turner S R and Daly R C 1988 J. Chem. Edu. 65322

Tyman J H P 1979 Chem. Soc. Revs. 8499

Uchino S, Tanaka T, Ueno T and Iwayanagi T $1991 \mathrm{~J}$. Vac. Sci. Technol. B9 3162

Ueno T 1998 Microlithography: Science and technology (eds) J R Sheats and B W Smith (USA: Marcel Dekker) p. 429

Voigt Anja 1999 Ph.D. Thesis, Humboldt University, Berlin Wallraff G M and Hinsberg W D 1999 Chem. Rev. 991801

Zöllinger H 1994 Diazo chemistry I: Aromatic and heterocyclic compounds (New York: VCH Publishers) p. 285 